\title{
COVID-19 and Renal Failure: Challenges in the Delivery of Renal Replacement Therapy
}

\author{
Sreedhar Adapa ${ }^{\mathrm{a}, 1}$, Narothama Reddy Aeddula ${ }^{\mathrm{b}}$, Venu Madhav Konala ${ }^{\mathrm{c}}$, Avantika Chenna ${ }^{\mathrm{d}, \mathrm{e}}$, \\ Srikanth Naramala ${ }^{\mathrm{f}}$, Bhaskar Reddy Madhira ${ }^{\mathrm{g}}$, Vijay Gayam ${ }^{\mathrm{h}}$, Mamtha Balla ${ }^{\mathrm{i}}$, \\ Vijayadershan Muppidij, Subhasish Bose ${ }^{\mathrm{k}}$
}

\begin{abstract}
Coronavirus disease 2019 (COVID-19), caused by severe acute respiratory syndrome coronavirus 2 (SARS-CoV-2), first officially reported in December 2019 in Wuhan City, Hubei province, China, and has since lead to a pandemic. Most cases result in minor symptoms such as cough, fever, sore throat, myalgia, fatigue, nausea, diarrhea, loss of smell, and abdominal pain. As of April 8, 2020, more than $1,485,000$ cases of COVID-19 have been reported in more than 200 countries and territories, resulting in over 90,000 deaths. Outcomes are worse in elderly patients, particularly males, and those with comorbidities, but can affect any age group. The incidence of acute kidney injury in patients with COVID-19 infection is about 3-15\%; and in patients with severe infection requiring care in the intensive care unit, the rates of acute kidney injury increased significantly from $15 \%$ to $50 \%$. Acute kidney injury is an independent risk factor for mortality in COVID-19 patients. The nephrologists, as well as intensivists, are facing immense daily challenges while providing care for these patients in the inpatient setting as well as end-stage renal disease pa-
\end{abstract}

Manuscript submitted April 9, 2020, accepted April 17, 2020

aDivision of Nephrology, Department of Internal Medicine, Adventist Medical Center, Hanford, CA 93230, USA

bDivision of Nephrology, Department of Internal Medicine, Deaconess Health System Inc, Evansville, IN, USA

'Division of Medical Oncology, Department of Internal Medicine, Ashland Bellefonte Cancer Center, 122 St Christopher Dr, Ashland, KY 41169, USA

dPhoebe Putney Memorial Hospital, 417 W 3rd Avenue, Albany, GA 31701, USA

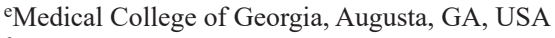

fDivision of Rheumatology, Department of Internal Medicine, Adventist Medical Center, Hanford, CA 93230, USA

gDepartment of Hematology and Oncology, Sunny Upstate Medical University, Syracuse, NY, USA

hDepartment of Medicine, Interfaith Medical Center, Brooklyn, NY, USA

iDepartment of Internal Medicine, University of Toledo and Promedica Toledo Hospital, Toledo, OH 43606, USA

${ }^{j}$ Department of Internal Medicine, IU Health Ball Memorial Hospital, Muncie, IN 47303, USA

k'Division of Nephrology, Lynchburg Nephrology Physicians, Lynchburg, VA, USA

${ }^{1}$ Corresponding Author: Sreedhar Adapa, Division of Nephrology, Adventist Medical Center, Hanford, CA 93230, USA. Email: sreedharadapa@gmail.com

doi: https://doi.org/10.14740/jocmr4160 tients on chronic dialysis in both inpatient and outpatient settings. In the current review article, we discussed the epidemiology and etiology of acute kidney injury, management of acute kidney injury including renal replacement therapy options (both hemodialysis and peritoneal dialysis) for inpatient floor, as well as intensive care unit settings. We also discussed the challenges faced by the outpatient dialysis units with COVID-19 infection. We discussed measures required to limit the spread of infection, as well as summarized the guidance as per the Centers for Disease Control and Prevention (CDC), American Society of Nephrology (ASN), American Society of Diagnostic and Interventional Nephrology (ASDIN) and the Vascular Access Society of the Americas (VASA).

Keywords: COVID-19; Kidney impairment; Acute kidney injury; Renal replacement therapy; Hemofiltration; Hemodialysis; End-stage renal disease; Peritoneal dialysis

\section{Introduction}

Coronavirus disease 2019 (COVID-19), caused by severe acute respiratory syndrome coronavirus 2 (SARS-CoV-2), first officially reported in December 2019 in Wuhan City, Hubei province, China, and has since lead to a pandemic. Most cases result in minor symptoms such as cough, fever, sore throat, myalgia, fatigue, nausea, diarrhea, loss of smell, and abdominal pain [1-3]. However, some patients progress to severe viral pneumonia, multiorgan failure, including respiratory failure from acute respiratory distress syndrome (ARDS), myocarditis, advanced renal failure, and death $[4,5]$. COVID-19 is a rapidly evolving disease, causing increased morbidity and mortality, and has been spreading quickly at an alarming rate globally. The limited knowledge of the SARS-CoV-2 natural history, asymptomatic carriers, intercontinental travelers, lack of effective treatment, potential reinfection, and overburdened global health systems are some of the critical elements involved in poor outcomes from COVID-19. As of April 8, 2020 more than 1,485,000 cases of COVID-19 have been reported in more than 200 countries and territories, resulting in over 90,000 deaths $[6,7]$. Outcomes are worse in elderly patients, particularly males, and those with comorbidities, but can affect any age group. 
Various studies have suggested an incidence of 3-15\% of acute kidney injury (AKI) in patients with COVID-19 infection $[8,9]$. In patients with severe COVID-19 infection in intensive care unit (ICU), the rates of AKI increased significantly to $14.5-50 \%[9,10]$. Based on the review of literature, we think AKI with COVID-19 is not a specific clinical feature, and it is a part of multi-organ failure with patients admitted to ICU. Regarding the incidence of AKI in other viral infections such as H1N1, AKI was reported in approximately $18 \%$ of the patients in a study of 661 critically ill patients infected with H1N1 influenza. Pettila et al reported that the incidence of AKI was $34 \%$ in 628 patients infected with H1N1 who are critically ill [11, 12]. COVID-19 infection is associated with an increased incidence of albuminuria and hematuria [13]. Notably, AKI is an independent risk factor for hospital mortality in COVID-19 patients $[13,14]$.

The nephrologists and critical care clinicians are facing daily challenges when providing care for patients with AKI requiring renal replacement therapy (RRT) from COVID-19 in the inpatient settings, and nephrologists face challenges in strategizing care for established end-stage renal disease (ESRD) patients on chronic dialysis in the hospital and outpatient settings.

Thus, we reviewed the available literature on the nature of AKI caused by COVID-19, as well as the available guidelines and strategies regarding the RRTs for the AKI and the hospitalized ESRD patients.

\section{AKI in COVID-19}

\section{Epidemiology and etiology of AKI}

Cheng et al described the incidence of AKI associated with COVID-19 and related mortality in 701 hospitalized patients from a single medical center in Wuhan City, China. Although the incidence of AKI was $5.1 \%$, there was a dose-dependent relationship between the degree of AKI and mortality. The incidence of proteinuria on admission was $44 \%$, and hematuria was $27 \%$. Whether proteinuria and hematuria were due to AKI or underlying chronic kidney disease (CKD) is unclear [14].

Similar studies from China also supported the strong correlation between AKI and risk of mortality (about 5.3 times higher in COVID-19 cases with AKI than without AKI). Observational data showed other signs of kidney dysfunction in COVID-19 patients, such as proteinuria in 59\% and hematuria in $44 \%$ hospitalized COVID-19 patients [13].

Arentz et al described the clinical characteristics and outcomes in 21 critically ill patients with COVID-19 in Washington State, USA. Two were on dialysis. The mortality was higher in this study, but the specific outcomes of the dialysis patients have not been described [15]. The clinical inpatient experience from Montefiore Medical Center, Bronx, New York, revealed that 109 patients developed AKI, and among them, 69\% were males. Forty-six percent of the patients needed ICU admission; and among them, $45 \%$ required RRT. The patients with AKI on CKD were discharged needing outpatient dialysis. The mortality was $31 \%$ on limited time follow-up. Forty-seven prevalent hemodialysis (HD) patients required inpatient admission for COVID-19, and the mortality among them was $8.5 \%$. The data were from the limited time follow-up in March 2020 [16].

The exact pathophysiology of kidney failure in COVID-19 is unclear. The leading cause of AKI in COVID-19 seems to be acute tubular necrosis driven by sepsis, multi-organ failure, and shock. Besides, SARS-CoV-2 binds with angiotensin-converting enzyme 2 (ACE2) receptor, which is expressed abundantly in the renal podocytes and proximal tubular epithelial cells [17]. Recently, Zhong's lab in Guangzhou, China has successfully isolated SARS-CoV-2 from the urine sample of an infected patient [18]. Therefore, ACE2 could serve as a binding site, and play a role in potential renal injury mechanisms. Other postulated mechanisms include sepsis leading to cytokine storm syndrome or direct cellular injury from the virus.

\section{Management of AKI}

The current treatment of COVID-19 with AKI includes general and supportive management and RRT when necessary. There is no proven antiviral therapy available at present. In patients with severe AKI, initiation of RRT is indicated for traditional reasons such as uremia, uremic pericarditis, electrolyte imbalance such as hyperkalemia and metabolic acidosis, and hypervolemia, among others. Volume overload in the presence of ARDS can be detrimental and may make ventilator support suboptimal. Therefore, relative early initiation of RRT may be considered in COVID-19 patients with severe ventilatordependent respiratory failure (VDRF) for ARDS and concomitant volume overload.

\section{RRT Options for AKI or ESRD Patients With COVID-19 in Hospital}

Currently, there is no data to support the early initiation of RRT in COVID-19 patients with AKI. Established medical management such as a trial of high-dose loop diuretics for oliguria and hypervolemia, judicious use of balanced crystalloid intravenous fluids for prerenal state and intravascular expansion, should be employed. In the patients who developed AKI from COVID-19 infection, around $1.5-9 \%$ of them required RRT in the form of continuous renal replacement therapy (CRRT) [19, 20]. The proportion of patients needing RRT increased to 5.6$23 \%$ with severe infection [21, 22].

In COVID-19 patients with severe AKI and with established ESRD, the continuation of their RRT is vital for survival. We will focus our discussion here on modalities of RRT that can be used in appropriate situations for patients with COVID-19 with severe AKI or ESRD.

\section{CRRT}

Based on the clinical practice patterns, the primary indication for choosing CRRT over intermittent HD is hemodynamic instability and high catabolic states. Though there are no rand- 
omized trials, CRRT is widely believed to be associated with less hemodynamic instability given the rates of fluid and solute removal are slower than with intermittent HD [23]. Unfortunately, CRRT is not available in all acute health care settings.

The different modalities of CRRT are defined by the main mechanism with which clearance is achieved: simple diffusion (continuous venovenous hemodialysis (CVVHD)), convection (continuous venovenous hemofiltration $(\mathrm{CVVH})$ ), or a combination of both (continuous venovenous hemodiafiltration (CVVHDF)). Many intensivists and nephrologists prefer to use CVVH in the belief that pure convection will remove a higher number of larger molecules than diffusion-based CVVHD. Others argue that CVVHD is easier and, given the lack of comparative evidence, prefer this. There is a third school, who favors CVVHDF banking on both convective and diffusion principles for solute clearance and ultrafiltration [24].

In severe ARDS, these modalities can be used in conjunction with extracorporeal membrane oxygenation (ECMO) as and when indicated [25]. Because of hemodynamic instability and minimization of nursing staff exposure, CRRT has been recommended by the American Society of Nephrology (ASN) experts as one of the recommended RRT in COVID-19 patients with severe AKI [26].

Moreover, high-volume hemofiltration at a dose of $6 \mathrm{~L} / \mathrm{h}$ has been shown to remove inflammatory cytokines (interleukin-6) and improved the Sequential Organ Failure Assessment scores at day 7 in patients with sepsis [27]. Therefore, CRRT may play a role in patients with COVID-19 and sepsis syndrome.

\section{CRRT dosing}

In general, Kidney Disease: Improving Global Outcomes (KDIGO) guidelines for CRRT dosing can be followed [28]. If using pre-filter dilution, it is recommended to use $25-30$ $\mathrm{mL} / \mathrm{kg} / \mathrm{h}$; and for post-filter dilution, $20-25 \mathrm{~mL} / \mathrm{kg} / \mathrm{h}$ is recommended. When using high-volume hemofiltration in cases with severe sepsis, recommended clearance is $>35 \mathrm{~mL} / \mathrm{kg} / \mathrm{h}$ to help to clear inflammatory mediators. Experts have suggested certain modifications to the dialysis delivery for COVID19-positive patients during current pandemic such as: use of extra-long tubing to allow for staff to access machine outside the glass doors in ICU, high dialysate flow of $30-40 \mathrm{~mL} / \mathrm{kg} / \mathrm{h}$ and 8-h treatment time per patient per day to ensure optimum use of limited resources. Once initiated on CRRT, the treatment modifications for prescription change can be performed by the well-trained ICU nurse taking care of the critically ill patient saving valuable dialysis nursing time.

\section{Prolonged intermittent renal replacement therapy (PI- RRT)}

PIRRT is a concept of hybrid treatment modality that provides RRT for an extended period (usually $6-18 \mathrm{~h}$ ) but is intermittent (at least three times per week). The principle of PIRRT includes amalgamation of both convective (i.e., hemofiltration) and diffusion (i.e., HD) principles, depending on the method of solute removal [29]. Other terms used to describe PIRRT include sustained low-efficiency daily dialysis (SLEDD), sustained low-efficiency daily diafiltration (SLEDD-f), extended daily dialysis (EDD), slow continuous dialysis (SCD), go slow dialysis, and accelerated venovenous hemofiltration (AVVH) or hemodiafiltration. PIRRT can be performed on most machines that are used for standard intermittent HD. Specific CRRT machines can also be used for PIRRT with some modification of treatment prescriptions. Machines used for PIRRT should have the capability to run at low blood and dialysate flow rates. Some machines are designed specifically for PIRRT, and have specific capabilities for solute removal by convection, diffusion, or both [18]. Standard extracorporeal circuit tubing and hemodialyzers are used for PIRRT. High-flux dialyzers are usually utilized for PIRRT as others may result in lower clearance. High-cutoff dialyzers, which are studied in PIRRT, use a membrane that allows clearance of larger solutes compared with high-flux membranes. PIRRT with high-cutoff membranes may provide better clearance of middle molecules, but the clinical significance of this is not known [30].

Slow continuous ultrafiltration (SCUF) may be used to treat isolated cases with fluid overload. SCUF by itself is not useful in patients with metabolic abnormalities, because solute removal is minimal. Therefore, SCUF will not be an acceptable RRT in COVID-19 positive patients with severe AKI.

PIRRT can be used in hospitals where CRRT is not available. PIRRT can help optimize the dialysis nurses' time and exposure risk, as multiple patients could be dialyzed simultaneously over a period by one nurse. PIRRT is particularly helpful and could be preferred over intermittent HD as higher ultrafiltration volume can be achieved without significant hemodynamic instability. The data on the hemodynamic stability during hybrid treatments are from a randomized, controlled trial that compared CRRT with PIRRT among 39 critically ill patients. There was no significant difference between treatments in inotrope dose or number [31]. Unlike CRRT, this modality will require a designated dialysis nurse to be present for the whole duration of therapy, but one nurse could do PIRRT on more than one patient in adjacent ICU rooms simultaneously.

\section{PIRRT dose}

Generally, a recommended dose of PIRRT would be $20 \mathrm{~mL} /$ $\mathrm{kg} / \mathrm{h}$ per $24 \mathrm{~h}$ for a patient, and the total treatment then can be divided by the number of hours per treatment, which is typically $10 \mathrm{~h}$ or less [26].

\section{Conventional intermittent hemodialysis (IHD)}

In COVID-19-positive patients who need initiation of RRT for severe AKI and are hemodynamically stable, IHD can be performed. In general, patients with severe AKI in COVID-19 are critically ill and at higher risk of death. These patients with AKI will likely in general have hemodynamic instability making it challenging to deliver IHD. However, patients with 
pre-existing advanced CKD may be more prone to develop the ESRD with COVID-19 infection-related sepsis mediated AKI. These patients, if hemodynamically stable, could be initiated on IHD on an elective basis, if indicated. However, this modality would require a HD nurse to be present with the patient for the entire duration of treatment. However, this would be the modality of choice for hemodynamically stable patients with severe AKI on the general hospital floor needing RRT.

\section{IHD dose}

Considering optimal utilization of resources during the COVID-19 pandemic, we need to be receptive to modification of typical IHD prescription for an individual patient. Patients may continue three times/week Kt/V urea 1.3/treatment standard IHD per KDIGO guideline, but not every patient needs 4-h IHD to achieve that; and we should consider the shortest duration that achieves the metabolic and volume control. Care should be taken to minimize 1:1 dialysis nurse time in the room.

\section{Peritoneal dialysis (PD)}

PD may not be considered as the first choice of RRT in case of severe AKI in COVID-19 patients. Urgent-start PD requires a dedicated team of experts in an institution that includes general surgeon or interventional radiologist/nephrologist inserting PD catheter, and a team well versed with urgent-start PD. Not many hospitals would have these personnel or resources, and there will be a questionable level of comfort around PD in the critical care environment.

However, given the pandemic, urgent-start PD can be an option where traditional resources or capacity for acute dialysis with CVVHD/IHD has been exceeded. The unique advantages of $\mathrm{PD}$ are many. It does not require significant infrastructure, which is a concern in many low/middle-income countries, and is relatively cost-effective. It reserves limited central vascular access sites for other purposes for critically ill patients and no need for systemic anticoagulation. Moreover, utilization of cycler PD can lead to the minimization of dialysis nursing exposure for the use of RRT in these patients.

There are multiple concerns about the effectiveness of PD as RRT in critically ill COVID-19 patients with multi-organ failure. The ultrafiltration rate with PD is unpredictable and the degree of solute clearance can fall short in these patients with the hypercatabolic state. There are also concerns about mechanical failure and the risk of peritonitis related to PD catheter in these patients. Lastly, there could be detrimental effects of PD on respiratory status in patients on a mechanical ventilator, as a significant volume of PD fluid in peritoneal cavity can impair diaphragmatic excursion, especially in those needing prone ventilation [26].

If established urgent-start PD program is already not in place in an institution, it would be generally advisable not to embark on putting resources for this purpose during this time of great stress on health care systems. In the case of estab- lished PD patients, we should try to keep on PD through critical illness. Otherwise, we may need to change RRT modality to CRRT, PIRRT, or IHD if it is ineffective, cumbersome, or difficult to deliver in the ICU setting. This would require vascular access placement and continuation of routine care for the PD catheter, which may be left in place for future use.

\section{PD dose}

Lower dwell volume such as $1 \mathrm{~L}$ should be used in urgent-start PD 24 - $48 \mathrm{~h}$ after insertion of PD catheter. Then the volume may be gradually increased over time to minimize the risk of mechanical complications. Although either manual exchanges or cycler-based PD can be used in AKI, manual PD may be challenging if significant ultrafiltration is warranted. Manual exchanges may be more comfortable if there is slow inflow and outflow. Cycler-based PD may be used based on availability and nursing expertise. It can be helpful to handle high-volume hourly exchanges with cycler continuously for critically ill intubated patients to minimize the ill effect on mechanical ventilation. Higher dextrose solution $(2.5 \%$ or $4.25 \%)$ should be used if urgent fluid removal is required. Medication dosing with continuous PD is not well studied but CRRT literature can guide in this matter.

\section{General measures recommended for RRTs}

It is recommended that: 1) Health-care personnel (HCP) should follow what the CDC recommended of personal protective equipment (PPE) and safety guidelines during their interactions with the patients; 2) Nephrologists should collaborate with ICU teams and primary teams to rely on physical examination findings to minimize patient contact. Telehealth can be instituted when available; 3) Indications for RRT are similar to other patients with AKI, and providers with significant expertise should place dialysis catheters $[31,32]$.

\section{RRT recommendations for patients in ICU}

It is stated that: 1) The critically ill COVID-19-positive patients should be cohorted in isolation ICUs dedicated to COVID-19; 2) The preferred dialysis modality is CRRT or PIRRT, if available; 3) CRRT is preferred over IHD in the setting of isolation, as IHD needs 1:1 nursing support; 4) ICU nurses should be competent in the use of CRRT, limiting exposure of HD nurses; 5) The CRRT machine is set up in a way that predialysis preparation and troubleshooting can be done outside the patient's room, thus minimizing the exposure and limiting the use of PPE; 6) IHD can also be performed with close monitoring and management of hemodynamics if CRRT and PIRRT are not available; 7) If the patients needing CRRT exceeds the machines available, prolonged intermittent treatments (e.g., 10 $\mathrm{h}$ instead of continuous) with higher flow rates (e.g., $40-50$ $\mathrm{mL} / \mathrm{kg} / \mathrm{h})$ and after terminal cleaning, can be used for another patient [32]. 


\section{RRT recommendations for patients in general hospital floors}

It is recommended that: 1) Hemodynamically stable COVID-19 patients should be cohorted on a designated hospital floor; 2) Patients with active or suspected COVID-19 needing dialysis treatment should not be transported to a central acute dialysis unit even if they are hemodynamically stable; 3) If all the COVID-19 patients are isolated on one floor, one dialysis nurse may be able to monitor two or three patients during IHD if dialysis patients are cohorted in adjacent rooms, and video/ electronic monitoring is available. The nurse will keep on full PPE for the entire duration in antechamber room adjacent to two/three isolation rooms with patients on IHD. The dialysis nurse will only enter the patient room if the patient needs anything or to troubleshoot the dialysis machine; 4) The peritoneal dialysis can be continued on selected acute-start patients or established PD patients as cycler-based automated peritoneal dialysis (APD) to limit the exposure of dialysis nursing staff [32].

\section{Vascular access for RRT}

For AKI patients requiring initiation of RRT in the form of HD or a variation of it, availability of functional vascular access is essential. HD catheters are usually put in by nephrologists, interventional radiologists, vascular surgeons or intensivists. During the pandemic, additional providers can be recruited to place vascular catheters. Catheter lengths are significant to ensure adequate blood flow and reduce clotting. When trying to establish new vascular access, it is recommended to use temporary dialysis catheters in the right internal jugular (IJ) or subclavian (SC) vein followed by femoral venous access. The length of the catheter for the right IJ access should be $15 \mathrm{~cm}$. For femoral, left IJ, and SC vein catheters, the length should be around $24-30 \mathrm{~cm}, 20 \mathrm{~cm}$, and $20 \mathrm{~cm}$, respectively. It is recommended to use double-layered protection for the personnel performing the procedure.

For COVID-19 patients established as ESRD or advanced CKD patients who need to initiate dialysis in hospital, preexisting arteriovenous fistula (AVF) or arteriovenous graft (AVG) may be used. However, these accesses would be preferably used for hemodynamically stable patients who can tolerate IHD. Although AVF/AVG can be used for SLED/PIRRT, the concern of needle dislodgement and exsanguination is real. Video monitoring, 1:1 nursing care, and reinforced taping of needles can be helpful in such a scenario. If institutional norms or guidelines are contradictory, it is preferred to have a temporary HD catheter for RRT.

\section{Anticoagulation}

Anticoagulation for CRRT/PIRRT may be essential in COVID-19 patients with severe AKI. ESRD patients on IHD also need routine anticoagulation. When choosing anticoagulation, it is crucial to assess the patient's coagulation risk profile. Pa- tients with severe COVID-19 infection having high bleeding risk with international normalized ratio $>1.5$ or thrombocytopenia, it is recommended to avoid anticoagulation. In normal or hypercoagulable states, one can use heparin or citrate-based anticoagulation depending on hospital protocols. Per KDIGO guidelines, regional citrate anticoagulation rather than heparin is recommended when there are no contraindications like liver failure, lactic acidosis, and severe shock. The usual target PTT for unfractionated heparin infusion in CRRT is $60-80 \mathrm{~s}$. The dose adjustments can be made per standard protocols. It is advisable not to use a citrate-based anticoagulation protocol if one is already not in place and practiced in an institution. When using CRRT in combination with ECMO, anticoagulation is not needed as ECMO utilizes systemic heparinization.

\section{Care and disinfection of RRT equipment in hospital}

It is required that: 1) The dialysis equipment should be cleaned with the disinfectant before removing it from the room; 2) Hospital infection control and policy directs the proper disposable of RRT machine equipment; 3) As per the hospital protocol, CRRT filters need to be changed every $72 \mathrm{~h}$ or longer.

\section{Strategies to ensure optimized use of dialysis resources in hospital}

The strategies include: 1) In cases of AKI in patients under investigation (PUI), delay initiation of RRT if possible, in case COVID-19 test results are expected in $24-48 \mathrm{~h}$. This will avoid overutilization of RRT and will conserve PPE which is already in short supply; 2) For volume overload and hyperkalemia, utilize loop diuretics and potassium binders to delay the need for RRT initiation; 3) Consider CRRT replacement fluid solution rate of $15 \mathrm{~mL} / \mathrm{kg} / \mathrm{h}$ once metabolic control is achieved to conserve CRRT solutions; 4) The pharmacy can make essential CRRT solutions if branded CRRT solutions are in short supply; 5) Conventional HD machine can be used to do PIRRT with modification of HD prescription; 6) Adopt creative CRRT/PIRRT maneuvers to minimize exposure of COVID-19 to dialysis nurses. For example, setting up and keeping CRRT/HD machines outside the patient's room with bloodlines going through the ICU door in a negative pressure room; 7) Multidisciplinary rounding using video conferencing for ICU patients daily; 8) Early establishment and continued discussion around goals of care for critically ill COVID-19 patients to ensure appropriate allocation of resources; 9) While it is imperative to optimize resource utilization and minimize exposure to healthcare providers, we must not compromise the safety of patients.

\section{Non-RRT extracorporeal therapies in COVID-19 patients}

Apart from need for RRTs in severe AKI, there are modalities for extracorporeal blood purification in COVID-19 patients that are being utilized for experimental treatment such as: cytokine adsorber and plasma exchange or infusion of convalescent 
plasma. In severe COVID-19 cases with hypoxemic respiratory failure despite optimal ventilator support, ECMO can be used.

\section{Outpatient Dialysis and COVID-19 Infection}

COVID-19 infection poses a unique threat to dialysis patients, particularly the in-center HD patients, because of increased risk of cluster exposure and patients being in close proximity. Most are elderly and have multiple comorbidities. Studies have shown that the HD patients infected with COVID-19 infection had the milder disease, less intense lymphopenia, and lower levels of inflammatory cytokines compared to other patients with COVID-19 infection [33]. So far, no COVID-19 infection was reported in PD patients. The experience of COVID-19 in HD patients has been described below.

The initial impact on the dialysis patients comes from an experience of a single HD center in Renmin Hospital, Wuhan University, where $37 \mathrm{HD}$ out of $230 \mathrm{HD}$ patients and four out of 33 staff members developed COVID-19 infection between January 14 and February 17, 2020. A total of six patients died from COVID-19 infection [33].

Italian researchers described two dialysis centers during the COVID-19 outbreak from Lombardy. At a satellite HD unit with 60 patients, 18 were infected. These patients were immediately isolated and treated separately. The time-lapse between the first patient and the 18th is 1 week. One patient was in critical condition, and no healthcare staff has been infected [34]. At the second center, four out of 170 patients were infected. These patients were promptly isolated, and none of the staff were affected. The testing for COVID-19 was performed on the symptomatic patients [34].

Fu et al described a case of a 75-year-old male on maintenance HD with COVID-19. The patient received intermittent hemodiafiltration in the hospital for a total duration of $53 \mathrm{~h}$. The patient was treated with umifenovir, which is a membrane fusion inhibitor, and oseltamivir. The patient was discharged in day 15 after he was asymptomatic and radiological clearance with two negative polymerase chain reaction (PCR) tests [35].

Ferrey et al reported a case of a 56-year-old male on maintenance HD presented with atypical gastrointestinal symptoms initially and later diagnosed with COVID-19 associated ARDS. The patient was initiated on continuous CVVHDF. The patient required prone ventilation and ECMO. The patient was receiving hydroxychloroquine and tocilizumab, and the outcome was not reported. [36].

Watnick and the team have described the measures that were taken at the outpatient dialysis clinic to limit the spread when faced with first COVID-19 infection in the USA. This article and ASN webinar provided guidance for many nephrologists across the USA how to handle COVID-19 in the outpatient dialysis setting $[37,38]$.

\section{Role of medical director for an outpatient dialysis clinic}

The role of the medical director is very critical in preventing the transmission of COVID-19 infection at the outpatient di- alysis clinic and safeguarding both the dialysis patients and the care staff. The medical director should work in a close relationship with the medical staff/HCP and manager of the dialysis facilities to ensure that effective dialysis care continues [38]. The frequent presence of the medical director and attending meeting with staff at regular intervals will be reassuring for the care staff.

There should be a core team with a medical director, dialysis clinic manager, and charge nurse who should have clear communication at regular intervals. The conversation should be on the updates, guidelines, and action plans. This core team should effectively use the local resources, care services, and should be thoughtful of rapidly evolving scenarios [38]. The core team should be communicating and supporting the care staff and the patients at regular intervals. A strict no visitor policy should be implemented at the dialysis center when the patients are receiving dialysis.

The patients should come for dialysis as it is considered as a life-saving treatment even though situations like lockdown are in effect. The dialysis center should provide the letter stating the importance of getting treatment for the patients, staff, and transportation companies.

All the patients should be educated to stay at home when not receiving dialysis. All the family members living with dialysis patients should be trained on hand hygiene, self-monitoring for the symptoms, and prompt reporting of symptoms. Patients should be educated that young people can be vectors of the disease without developing symptoms, so the precautions should be taken to prevent transmission within the family. The patients are encouraged to use personal or designated transportation methods to the dialysis centers instead of using public transport.

All the staff should be educated on hand hygiene, universal precautions, disposal of the waste, and use of PPE. All the team should be updated on the epidemic and the latest care guideline recommendations. Precautions should be taken when performing aerosol generating procedures (AGP), which is not feasible at the outpatient dialysis centers and must be done in life-saving situations with proper PPE. PPE count should be conducted daily. The facility should have a policy and procedure in place to deal with the shortage of PPE and preserving PPE supply in times of scarcity [38, 39].

There should be a plan in place regarding providing dialysis for suspected patients with COVID-19 infection. The patients with confirmed COVID-19 infection should have a designated dialysis shift ideally. The asymptomatic patients with COVID-19 infection should be separated from symptomatic patients if they must get dialysis treatment at the same time. The symptomatic patients should be treated at the corner or end of the row station.

ASN and CDC have provided guidance on mitigating the risk of COVID-19 for dialysis centers [38, 39].

General precautions to limit the spread of COVID-19 infection to dialysis patients and care staff

These include: 1) Adequate education for the patients regarding COVID-19, and actions taken by the dialysis facility to protect them; 2) The patients should be educated on hand hygiene, respiratory hygiene, cough etiquette, and appropriate disposal of contaminated items; 3 ) The HCP should be pro- 
vided with information on COVID-19; 4) The HCP should be educated on infection prevention and control measures with emphasis on hand hygiene, selection, and/or use of PPE; 5) The dialysis unit waiting area should be prepared with signs posted on hand hygiene, respiratory hygiene, and instructions provided to the patients with fever and respiratory infection symptoms to alert the staff; 6) The waiting area should have tissues, alcohol-based hand rub, and trash cans; 7) The PPE should be easily accessible, and the alcohol-based sanitizers should be made available in waiting and treatment areas; 8) All the patients, visitors, and transport personnel should be screened for temperature and respiratory symptoms; 9) A questionnaire should be provided regarding symptoms, contact history, and travel history for all the patients at every shift of dialysis; 10) All the requests for transient dialysis treatments (visiting dialysis patients) should be discouraged and must get the approval of the medical director/unit director; 11) The waiting area should have space for ill patients to be separated from other patients by at least 6 feet; 12) The patients should be instructed to call the dialysis facility ahead of their arrival if they develop fever or respiratory symptoms so that they can be appropriately triaged; 13) The patients should wash their hands and fistula before starting the dialysis; 14) The waiting area, treatment floor of the dialysis center, and the vehicle should be sanitized/disinfected regularly.

\section{Staff precautions}

Some requirements are: 1) The HCP should not report to work when they are ill, and sick leave policies should be flexible; 2) The HCP should have active screening before their shift, and if they develop symptoms during work, they should wear a mask and return home; 3) PPE inventory should be accessible (facemasks, gowns, gloves, and eye protection); 4) The isolation gowns should be worn over or instead of the cover gowns and discarded in a dedicated container; 5) Eye protection should be either goggle or face masks. Contact lenses and personal glasses are not considered adequate eye protection; 6) Routine disinfection procedures are appropriate for COVID-19; 7) Active screening for temperature and symptoms of HCP before, during, and after shifts during the time of community transmission and if COVID-19 patients are in the facility.

\section{Dialysis patient with symptoms}

These steps should be followed: 1) The patient with symptoms should be appropriately triaged and directed to the hospital if appropriate, and the hospital should be informed about the arrival of symptomatic patients; 2) If the patients are getting transferred to another facility, the transport personnel should be notified; 3) If COVID-19 infection is suspected, the local health department should be notified; 4) If the patient is deemed to receive the treatment at the facility, the patient should be separated from other patients by at least 6 feet and wear a mask; 5) Symptomatic patients should be dialyzed in a separate room with the door closed if available ideally; 6) Hep- atitis B isolation rooms should be used for the patients with symptoms if the patient is hepatitis B surface antigen-positive or if the facility has no patients with hepatitis B infection on the census; 7) Patients with different etiologies should not be cohorted if the etiology of respiratory symptoms is known; 8) If more than one patient has respiratory symptoms at the dialysis facility, the patients should be cohorted at the section of the dialysis clinic and/or on the same shift (ideally last shift of the day).

\section{Dialysis patients with suspected or confirmed COVID-19 infection}

General measures include: 1) There should be designated cohort dialysis facility assigned in the geographical area to treat patients with suspected or confirmed COVID-19 infection; 2) If designated dialysis facility is not feasible, then isolation shifts should be created to dialyze these patients; 3 ) It is essential to isolate the patients with suspected COVID-19 from the patients with confirmed infection; 4) The staff should wear PPE, and necessary precautions should be taken.

\section{Transportation aspects}

The instructions are: 1) Patients should be encouraged to use private transportation if symptomatic or suspected to have COVID-19, or when feasible; 2) The drivers who are transporting suspected or confirmed COVID-19-positive patients should be informed, if they use the transport companies; 3 ) Suspected patients should wear the mask during the travel, and the drivers should report any patient with symptoms to the dialysis facility; 4) Drivers are encouraged to wear the mask and gloves when transporting the patients; 5) Hand sanitizers should be available for patients and drivers; 6) The drivers should be isolated if they have been exposed and the vehicle should be thoroughly sanitized when exposed; 7) Vehicles should be sanitized between the rides and should have thorough nightly deep cleans [40].

\section{Vascular access procedures}

The vascular interventions should be done promptly, as they are the life-saving procedures. Access interventions should be done in an ambulatory setting rather than a hospital setting because of the decreased risk of exposure. Outpatient access intervention allows patients to receive dialysis uninterruptedly and alleviate new interventions and unnecessary hospital admissions. The new vascular access placement, like arteriovenous fistula/graft (AVF/AVG), PD catheters should continue as planned [41].

\section{Unforeseen issues}

The dialysis centers may face many challenges during this 
pandemic, so there should be a policy in place to deal with unforeseen circumstances. The medical director and the core team should communicate regularly to deal with these issues. There could be staffing challenges because of quarantining from the exposure. There could be increased incident dialysis patients who develop AKI from COVID-19 needing RRT, failed renal transplants and switching dialysis modality. If the number of dialysis patients overwhelms the capacity of the dialysis clinic, extra shifts should be added, and modifications of dialysis prescriptions should be done. Issues in transportations can also play a significant role, and sometimes patients can miss dialysis. The patients should be educated to limit the fluid, potassium intake and have potassium-binding agents at hand in the event when they miss dialysis. The patients are discouraged from going to the hospital to limit the exposure if they miss dialysis, and every effort should be made to accommodate them for dialysis treatment.

\section{Patients receiving home dialysis}

The home dialysis patients should be educated on the COVID-19 infection. They should regularly monitor for fever and respiratory symptoms at home and contact the home dialysis nurse if develop any symptoms. The patients should be educated on precautions to avoid contact with persons who might be exposed and practice social distancing. The patients should use telehealth for medical advice and doctor visits.

Home dialysis patients needing labs drawn, erythropoietin injections, or assessments, they all should be combined to a single visit to limit the exposure. The patients are encouraged to order surplus dialysis supplies because of concerns associated with the supply chain. There are also concerns regarding the timely interventions of access in home dialysis patients. Proper installation or repair of PD catheters; interventions of AVF/ AVG should be done as they are life-saving procedures. Every effort should be made to initiate patients on home dialysis as it limits the exposure compared to in-center HD. Urgent-start PD should be encouraged.

\section{Treatment}

There are many variable factors when administering medications to dialysis patients. The molecular size, volume of distribution, and protein binding affinity are the key issues to consider. Most of the trials exclude CKD patients from the trials [40]. Food and Drug Administration (FDA) has not approved any specific drug for the treatment of COVID-19. Several clinical trials are underway across the globe, investigating a multitude of drugs. Remdesivir, lopinavir/ritonavir, chloroquine/ hydroxychloroquine, darunavir/ritonavir, favipiravir, tocilizumab, colchicine, and azithromycin, which are approved for other indications, are being used for treating COVID-19. Spike protein of SARS-CoV2 enters the human cell through the ACE2 receptor aided by type II transmembrane serine protease (TMPRSS2), and chloroquine acts by interfering with ACE2. Chloroquine decreases the severity of COVID-19 pneumonia as per the experience in the treatment of 100 patients [42]. The nephrologists should pay close attention to the dosing and safety of these drugs in the setting of renal failure and dialysis.

\section{Conclusions}

COVID-19 is a global pandemic that has resulted in a rapid spread of infection with an increase in morbidity and mortality worldwide. AKI is one of the common complications with COVID-19, and is associated with increased morbidity and mortality. RRTs in the health care facilities pose unique challenges in terms of shortage of staff and machines. The risk of exposure is also high while providing RRT. The risk of infection is also high in the outpatient dialysis setting because of clustering. Outpatient dialysis poses many obstacles with staffing, transportation, and risk of exposure when encountered with COVID-19 patients.

\section{Acknowledgments}

None to declare.

\section{Financial Disclosure}

None to declare.

\section{Conflict of Interest}

None to declare.

\section{Author Contributions}

All authors contributed equally. All the authors reviewed the manuscript and agreed with the findings and interpretation.

\section{Data Availability}

The authors declare that data supporting the findings of this study are available within the article.

\section{Abbreviations}

COVID-19: coronavirus disease 2019; SARS-CoV-2: severe acute respiratory syndrome coronavirus 2; ARDS: acute respiratory distress syndrome; AKI: acute kidney injury; ICU: intensive care unit; ESRD: end-stage renal disease; CKD: chronic kidney disease; ACE2: angiotensin converting enzyme-2; RRT: renal replacement therapy; CDC: Centers for Disease Control and Prevention; WHO: World Health Organization; ASN: American Society of Nephrology; ASDIN: 
American Society of Diagnostic and Interventional Nephrology; VASA: Vascular Access Society of the Americas; VDRF: ventilator-dependent respiratory failure; CRRT: continuous renal replacement therapy; CVVHD: continuous venovenous hemodialysis; CVVH: continuous venovenous hemofiltration; CVVHDF: continuous venovenous hemodiafiltration; ECMO: extracorporeal membrane oxygenation; KDIGO: Kidney Disease Improving Global Outcomes; PIRRT: prolonged intermittent renal replacement therapy; SLEDD: sustained lowefficiency daily dialysis; SLEDD-f: sustained low-efficiency daily diafiltration; EDD: extended daily dialysis; SCD: slow continuous dialysis; AVVH: accelerated venovenous hemofiltration; SCUF: slow continuous ultrafiltration; IHD: intermittent hemodialysis; PD: peritoneal dialysis; PPE: personal protective equipment; APD: automated peritoneal dialysis; AVF: arteriovenous fistula; AVG: arteriovenous graft; PUI: patient under investigation; PCR: polymerase chain reaction; AGP: aerosol-generating procedures; HCP: health-care personnel; FDA: Food and Drug Administration

\section{References}

1. Coronavirus Disease 2019 (COVID-19) Symptoms. Centers for Disease Control and Prevention. United States. February 10, 2020. Archived from the original on January 30, 2020.

2. Q\&A on coronaviruses (COVID-19). World Health organization (WHO). Archived from the original on January 20, 2020. Retrieved March 11, 2020.

3. Hopkins Claire. Loss of sense of smell as marker of COVID-19 infection. Ear, Nose and Throat surgery body of United Kingdom. Retrieved March 28, 2020.

4. Hui DS, E IA, Madani TA, Ntoumi F, Kock R, Dar O, Ippolito G, et al. The continuing 2019-nCoV epidemic threat of novel coronaviruses to global health - The latest 2019 novel coronavirus outbreak in Wuhan, China. Int J Infect Dis. 2020;91:264-266.

5. Q\&A on coronaviruses. World Health Organization (WHO). Archived from the original on January 20, 2020. Retrieved January 27, 2020.

6. Coronavirus COVID-19 global cases by the Center for Systems Science and Engineering (CSSE) at Johns Hopkins University (JHU). ArcGIS. Johns Hopkins CSSE. Retrieved April 8, 2020.

7. Coronavirus Update (Live): 1,001,069 Cases and 51,378 Deaths from COVID-19 Virus Outbreak - Worldometer. www.worldometers.info. Retrieved April 2, 2020.

8. Cheng Y, Luo R, Wang K, Zhang M, Wang Z, Dong L, Li J, et al. Kidney disease is associated with in-hospital death of patients with COVID-19. Kidney Int. 2020.

9. Zhou F, Yu T, Du R, Fan G, Liu Y, Liu Z, Xiang J, et al. Clinical course and risk factors for mortality of adult inpatients with COVID-19 in Wuhan, China: a retrospective cohort study. Lancet. 2020;395(10229):1054-1062.

10. Zhang G, Hu C, Luo L, Fang F, Chen Y, Li J, Peng Z, et al. Clinical features and short-term outcomes of 221 patients with COVID-19 in Wuhan, China. J Clin Virol. 2020;127:104364.
11. Martin-Loeches I, Papiol E, Rodriguez A, Diaz E, Zaragoza R, Granada RM, Socias L, et al. Acute kidney injury in critical ill patients affected by influenza A (H1N1) virus infection. Crit Care. 2011;15(1):R66.

12. Pettila V, Webb SA, Bailey M, Howe B, Seppelt IM, Bellomo R. Acute kidney injury in patients with influenza A (H1N1) 2009. Intensive Care Med. 2011;37(5):763-767.

13. Li Z, Wu M, Guo J. Caution on kidney dysfunctions of 2019-nCoV patients. medRxiv 2020; published online Feb 12, 2020.

14. Cheng Y, Luo R, Wang K, Zhang M, Wang Z, Dong L, Li J, Yao Y, Ge S, Xu G. Kidney disease is associated with in-hospital death of patients with COVID-19. Kidney International. 2020.

15. Arentz M, Yim E, Klaff L, Lokhandwala S, Riedo FX, Chong M, Lee M. Characteristics and outcomes of 21 critically ill patients with COVID-19 in Washington State. Jama. March 19, 2020.

16. https://www.asn-online.org/ntds/resources/Webcast_2020_04_02_COVID-19.mp4.

17. Pan XW, $\bar{X} u \bar{D}$, Z̆hang H, Zhou W, Wang LH, Cui XG. Identification of a potential mechanism of acute kidney injury during the COVID-19 outbreak: a study based on single-cell transcriptome analysis. Intensive Care Med. 2020.

18. The team of Zhong Nanshan responded that the isolation of SARS-CoV-2 from urine remind us to pay more attention to the cleaning of individuals and families. Guangzhou Daily. 2020.

19. Wang D, Hu B, Hu C, Zhu F, Liu X, Zhang J, Wang B, et al. Clinical characteristics of 138 hospitalized patients with 2019 novel coronavirus-infected pneumonia in Wuhan, China. Jama. 2020

20. Chen N, Zhou M, Dong X, Qu J, Gong F, Han Y, Qiu Y, et al. Epidemiological and clinical characteristics of 99 cases of 2019 novel coronavirus pneumonia in Wuhan, China: a descriptive study. Lancet. 2020;395(10223):507513.

21. Yang X, Yu Y, Xu J, Shu H, Xia J, Liu H, Wu Y, et al. Clinical course and outcomes of critically ill patients with SARS-CoV-2 pneumonia in Wuhan, China: a single-centered, retrospective, observational study. Lancet Respir Med. 2020.

22. Guan WJ, Ni ZY, Hu Y, Liang WH, Ou CQ, He JX, Liu $\mathrm{L}$, et al. Clinical Characteristics of Coronavirus Disease 2019 in China. N Engl J Med. 2020.

23. Vinsonneau C, Camus C, Combes A, Costa de Beauregard MA, Klouche K, Boulain T, Pallot JL, et al. Continuous venovenous haemodiafiltration versus intermittent haemodialysis for acute renal failure in patients with multiple-organ dysfunction syndrome: a multicentre randomised trial. Lancet. 2006;368(9533):379-385.

24. Ricci Z, Ronco C, Bachetoni A, D'Amico G, Rossi S, Alessandri E, Rocco M, et al. Solute removal during continuous renal replacement therapy in critically ill patients: convection versus diffusion. Crit Care. 2006;10(2):R67.

25. Ronco C, Navalesi P, Vincent JL. Coronavirus epidemic: preparing for extracorporeal organ support in intensive care. Lancet Respir Med. 2020;8(3):240-241. 
26. https://www.asnonline.org/g/blast/files/ASN Webinar Hospital_Care_And Treatment_Options for COVID19 _Positive_Patients_with_ESKD_and_AKI_0 $\overline{4}_{-} .02 .2020 \mathrm{a}$. pdf.

27. Ghani RA, Zainudin S, Ctkong N, Rahman AF, Wafa SR, Mohamad M, Manaf MR, et al. Serum IL-6 and IL-1-ra with sequential organ failure assessment scores in septic patients receiving high-volume haemofiltration and continuous venovenous haemofiltration. Nephrology (Carlton). 2006;11(5):386-393.

28. https://kdigo.org/wp-content/uploads/2016/10/KDIGO2012-AKI-Guideline-English.pdf.

29. Edrees F, Li T, Vijayan A. Prolonged Intermittent Renal Replacement Therapy. Adv Chronic Kidney Dis. 2016;23(3):195-202.

30. Schmidt JJ, Hafer C, Clajus C, Hadem J, Beutel G, Schmidt BM, Kielstein JT. New high-cutoff dialyzer allows improved middle molecule clearance without an increase in albumin loss: a clinical crossover comparison in extended dialysis. Blood Purif. 2012;34(3-4):246-252.

31. Kielstein JT, Kretschmer U, Ernst T, Hafer C, Bahr MJ, Haller H, Fliser D. Efficacy and cardiovascular tolerability of extended dialysis in critically ill patients: a randomized controlled study. Am J Kidney Dis. 2004;43(2):342-349.

32. https://www.asnonline.org/g/blast/files/AKI_COVID19 Recommendations_Document_03.21.2020.pdf.

33. Ma Y, Diao B, Lv X, Zhu J, Liang W, Liu L, Bu W, et al. 2019 novel coronavirus disease in hemodialysis (HD) patients: Report from one HD center in Wuhan, China. medRxiv. 2020.

34. Rombola G, Heidempergher M, Pedrini L, Farina M,
Aucella F, Messa P, Brunori G. Practical indications for the prevention and management of SARS-CoV-2 in ambulatory dialysis patients: lessons from the first phase of the epidemics in Lombardy. J Nephrol. 2020;33(2):193196.

35. Fu D, Yang B, Xu J, Mao Z, Zhou C, Xue C. COVID-19 Infection in a Patient with End-Stage Kidney Disease. Nephron. 2020:1-3.

36. Ferrey AJ, Choi G, Hanna RM, Chang Y, Tantisattamo E, Ivaturi K, Park E, et al. A case of novel coronavirus disease 19 in a chronic hemodialysis patient presenting with gastroenteritis and developing severe pulmonary disease. Am J Nephrol. 2020:1-6.

37. Watnick S, McNamara E. On the frontline of the COVID-19 outbreak: keeping patients on long-term dialysis safe. Clin J Am Soc Nephrol. 2020.

38. https://www.asn-online.org/ntds/resources/Webcast 2020_03 11_COVID-19.mp4.

39. https://www.co c. gov/coronavirus/2019ncov/hcp/dialysis. html?CDC_AA_refVal=https $\% 3 \mathrm{~A} \% 2 \mathrm{~F} \% 2 \mathrm{Fwww} . \mathrm{cdc}$. gov $\% 2$ Fcoronavirus $\% 2$ F2019-ncov\%2Fhealthcarefacilit ies\%2Fdialysis.html.

40. http://www.nephjc.com/news/2020/3/23/covid-and-thekidney-dialysis-edition.

41. https://cdn.ymaws.com/www.asdin.org/resource/resmgr/ covid_19/Maintaining_lifelines_ASDIN_.pdf.

42. Balla M, Merugu GP, Patel M, Koduri NM, Gayam V, Adapa S, Naramala S, et al. COVID-19, modern pandemic: a systematic review from a front-line health care providers' perspective. Journal of Clinical Medicine Research. 2020;12(4):215-229. 